\title{
Transcatheter arterial embolization promotes liver tumor metastasis by increasing the population of circulating tumor cells
}

\author{
Zhu-Ting Fang ${ }^{1,2, *}$ \\ Guang-Zhi Wang ${ }^{1, *}$ \\ Wei Zhang' \\ Xu-Dong Qu' \\ Rong Liu' \\ Sheng Qian' \\ Liang Zhu' \\ Bo Zhou' \\ Jian-Hua Wang'
}

'Department of Intervention

Radiology, Zhongshan Hospital, Fudan

University, Shanghai, People's Republic

of China; ${ }^{2}$ Department of Intervention

Radiology, Provincial Hospital of

Fujian Province, Teaching Hospital

of Fujian Medical University, Fuzhou,

People's Republic of China

*Authors who have contributed equally to this article

\author{
This article was published in the following Dove Press journal: \\ OncoTargets and Therapy \\ 4 November 2013 \\ Number of times this article has been viewed
}

\begin{abstract}
Transcatheter arterial embolization (TAE) is widely used as an effective palliative treatment for hepatocellular carcinoma (HCC), and can prolong survival time. However, the high incidence of tumor recurrence and metastasis after TAE is still a major problem. Recent studies demonstrated that circulating tumor cells (CTCs) contribute to tumor metastasis. In this study, we tried to clarify whether the residual HCC after TAE can increase metastasis by increasing the number of CTCs. An orthotopic liver tumor model in the Buffalo rat was established using green fluorescent protein (GFP)-transfected HCC cell line, McA-RH7777. Two weeks after orthotopic liver tumor implantation, the rats underwent TAE treatment from the gastroduodenal artery. Iodized oil or saline was injected intra-arterially. Blood samples were taken on day $0,1,3,7,14$, and 21 for detection of CTCs after TAE treatment. We analyzed the number of CTCs and assessed the metastatic potential of surviving tumor cells in rats between TAE and control groups. Our results demonstrated that the metastatic colonies in the lung were significantly increased by TAE treatment. The number of CTCs was also significantly increased by TAE treatment from day 7 to day 21 . The expression of hypoxia-inducible factor (HIF)- $1 \alpha$ and epithelial-mesenchymal transition (EMT) marker proteins ( $\mathrm{N}$-cadherin and vimentin) was upregulated, but E-cadherin was downregulated after TAE treatment. In conclusion, the metastatic potential of residual HCC can be induced by TAE treatment in a rat liver tumor model, which involves the acquisition of EMT features and an increased number of CTCs.
\end{abstract}

Keywords: transcatheter arterial embolization, hepatocellular carcinoma, circulating tumor cells, epithelial-mesenchymal transition

\section{Introduction}

Hepatocellular carcinoma (HCC) is one of the most prevalent malignancies worldwide, especially in the People's Republic of China, where the chronic viral infection of hepatitis $\mathrm{B}$ virus (HBV) is hyperendemic and its incidence has continued to escalate in recent years. ${ }^{1}$ The first-line treatment for HCC patients is surgical procedures, such as liver resection or transplantation. Unfortunately, patients with HCC are usually diagnosed at the late stage so only a few patients can be effectively treated with curative surgery. Transcatheter arterial embolization (TAE) or transcatheter arterial chemoembolization has been widely performed as a palliative treatment for nonsurgical HCC, which can prolong the survival of HCC patients. ${ }^{2-4}$ However, the high incidence of tumor recurrence and metastasis after TAE is still a major problem. ${ }^{5,6}$ A higher rate of pulmonary metastasis following TAE treatment has been reported in HCC patients. ${ }^{5}$ Therefore, novel therapies are urgently needed to decrease tumor recurrence and metastasis after TAE therapy.
Correspondence: Jian-Hua Wang Department of Intervention Radiology, Zhongshan Hospital, Fudan University, Nol 80 Fenglin Road, Xuhui, Shanghai, People's Republic of China

Tel +86 02I 64041990 ext 3088 Email wang.jianhua@zs-hospital.sh.cn 
Biological deterioration after TAE has been reported recently. Hypoxia is an inevitable consequence after TAE. ${ }^{6}$ Therefore, some studies demonstrated that the expression of hypoxia-inducible factor (HIF)- $1 \alpha$ is elevated in tumor tissues after TAE and thus increases the metastatic potential of surviving tumor cells. ${ }^{7-10}$

Recently, accumulating evidence indicated that circulating tumor cells (CTCs) may deposit in multiple distant organs and increase metastasis and intrahepatic recurrence. ${ }^{11-16}$ Several researchers demonstrated that the number of CTCs is higher in patients with advanced $\mathrm{HCC}$, which may act as a potential prognostic marker in HCC patients. ${ }^{17-20}$ Some studies used the CTC number as an index to evaluate the response to different therapies in HCC patients. ${ }^{21} \mathrm{CTC}$ s play a major role in the initiation of metastasis and tumor recurrence. ${ }^{22}$ However, the relationship between the number of CTCs and TAE treatment has not been investigated.

In this study, we aimed to investigate whether TAE treatment promotes liver tumor metastasis through increasing CTCs in an orthotopic rat liver tumor model, and to explore the underlying mechanisms. This study will hopefully help to build a novel therapy to reduce liver tumor metastasis in HCC patients.

\section{Materials and methods}

\section{Cell culture}

The rat cell line McA-RH7777 (CRL-1601'TM, American Type Culture Collection, Manassas, VA, USA) was transfected with green fluorescent protein (GFP) by Genechem (Shanghai, People's Republic of China) and cultured in Dulbecco's Modified Eagle's Medium (DMEM; Hyclone, Herndon, VA, USA) containing 10\% fetal bovine serum (FBS; Gibco, Grand Island, NY, USA) and 1\% penicillin and streptomycin (Gibco) in a humidified incubator at $5 \% \mathrm{CO}_{2}$ and $95 \%$ humidified atmosphere air at $37^{\circ} \mathrm{C}$.

\section{Chemically induced hypoxia and analysis of cell morphology}

Hypoxia was induced by adding cobalt chloride $\left(\mathrm{CoCl}_{2}\right.$, $100 \mathrm{RM} / \mathrm{L}$ ) to the medium for 48 hours. The morphological characteristics of control and $\mathrm{CoCl}_{2}$-treated cells were compared under microscopy (Olympus, Tokyo, Japan).

\section{Cell invasion assay}

Cell invasion was carried out by transwell assay (Boyden chambers; Corning, Cambridge, MA, USA). $1 \times 10^{5}$ cells in serum-free DMEM were seeded on a Matrigel-coated membrane ( $8.0 \mu \mathrm{m}$ pore size) in the top chamber of the well.
DMEM containing 10\% FBS was added to the lower chamber of each well. After 48 hours under hypoxic or normoxic culture conditions, cells on the top membrane of the chamber were removed with a wet cotton swap, while the cells on the underside membrane of the chamber were stained with Giemsa (Sigma Chemical Co, St Louis, MO, USA). Invaded cells in three random fields were counted under microscope (×200 magnification, Nikon, Tokyo, Japan).

\section{Antibodies}

Monoclonal antibodies used in immunofluorescence, immunoblotting, and immunohistochemistry included mouse anti-rat E-cadherin (Abcam, Cambridge, MA, USA), rabbit anti-rat N-cadherin (BioWorld, Atlanta, GA, USA), rabbit anti-rat vimentin (BioWorld), rabbit anti-rat HIF-1 $\alpha$ (BioWorld), and mouse anti-rat glyceraldehyde 3-phosphate dehydrogenase (GAPDH; Abcam).

\section{Immunofluorescence}

The expression of E-cadherin, N-cadherin, and vimentin in control and $\mathrm{CoCl}_{2}$-treated cells was examined by immunofluorescence. Cells were grown on glass coverslips to $40 \%-50 \%$ confluence, and then fixed using 3.5\% paraformaldehyde at room temperature for 5 minutes, followed by $0.1 \%$ Triton X-100 treatment for 5 minutes. The fixed cells were washed and then blocked in $1 \%$ bovine serum albumin for 30 minutes. Incubations with primary monoclonal antibodies against $\mathrm{E}$-cadherin, $\mathrm{N}$-cadherin, and vimentin were carried out overnight at $4^{\circ} \mathrm{C}$. The cells were washed and incubated with anti-mouse and/or anti-rabbit tetramethylrhodamineisothiocyanate (TRITC)-conjugated secondary antibody (Jackson ImmunoResearch Laboratories, Inc., West Grove, PA, USA). Cells were counterstained with 4'-6-diamidino-2-phenylindole (DAPI) to visualize cell nuclei. After wash, coverslips were mounted onto slides and examined under fluorescence microscopy (Olympus).

\section{Experiment animals and establishment of animal model}

Buffalo rats (aged 7-8 weeks; weighing 220-250 g) were purchased from Charles River Laboratories and maintained under standard pathogen-free conditions in the Experimental Animal Research Center at Shanghai Zhongshan hospital, Fudan University, People's Republic of China. The experimental protocol was approved by the Institutional Ethics Committee. A metastatic model of HCC in rats was established by GFP-transfected McA-RH7777 cells. Briefly, McARH7777-GFP cells $\left(5 \times 10^{6}\right)$ were injected subcutaneously 
into the right thigh of each rat. When the subcutaneous tumor had grown to approximately $1 \mathrm{~cm}$ in diameter (approximately 4 weeks after injection), it was harvested and cut into small pieces of equal volume $\left(2 \times 2 \times 2 \mathrm{~mm}^{3}\right)$, and implanted into the left liver lobes of 42 different buffalo rats to induce HCC, as previously described..$^{6,23}$

\section{TAE procedure}

Tumor-bearing rats were randomly assigned into the TAE group or the control group. In the TAE group, rats with orthotopic tumors underwent the TAE procedure on day 14 of implantation. The tumor size was around $8 \mathrm{~mm}$ in diameter according to magnetic resonance imaging (MRI). The TAE procedure was carried out as previously described. ${ }^{6}$ Briefly, the rats were anesthetized intraperitoneally with $400 \mathrm{mg} / \mathrm{kg}$ trichloroacetaldehyde hydrate (Hushi, Shanghai, People's Republic of China). Through the median incision, the gastroduodenal artery was exposed and the distal side was ligated. The suture was revolved around the proximal gastroduodenal artery and tensed when Silastic tubing (SFM10350; SF Medical, Hudson, MA, USA) was inserted from the gastroduodenal artery incision (Figure 1). After that, iodized oil (lipiodol, Guerbet, France) (1:3 dilution with saline, $0.6 \mathrm{~mL} / \mathrm{kg}$, TAE group, $\mathrm{n}=21$ ) or an equal amount of saline (control group, $\mathrm{n}=21$ ) was injected slowly. The injection speed must be slow enough to ensure that the embolic agent (iodized oil) was delivered into the liver without reflux to other vessels. The proximal gastroduodenal artery was ligated after the procedure. The procedure was performed under a Nikon SMZ1500 dissection microscope (Nikon). The incision in the abdomen was closed with a 3-0 suture.

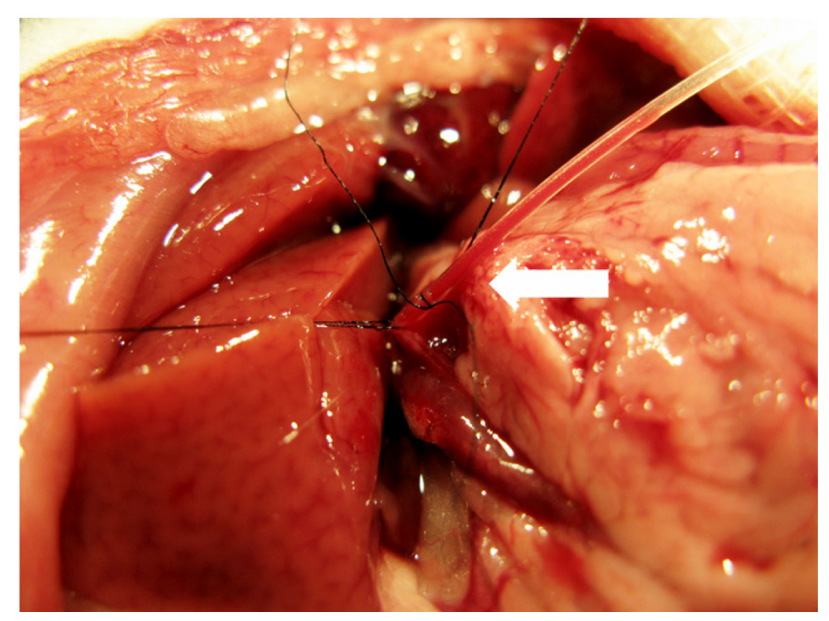

Figure I Catheterization and transcatheter arterial embolization were performed in a rat model. A silastic tube was inserted retrograde into the gastroduodenal artery (straight arrow), and iodized oil or saline was slowly injected.

\section{Imaging analysis and sample collection}

Blood samples were collected from the tail vein on day 0 (before TAE), 1, 3, 7, 14, and 21 after treatment for the detection of CTCs. To assess tumor growth and intrahepatic metastasis, MR images were acquired by $3.0 \mathrm{~T}$ magnetom (Avanto Trio, Siemens, Erlangen, Germany) with small animal coil. All animals underwent a T2-weighted imaging (T2 WI) on day 0,7 , and 14 after TAE with a multi-slice acquisition providing complete coverage of the entire liver. The tumor size was calculated as Volume $=\left(a \times b^{2}\right) / 2$, where ' $a$ ' is the widest diameter and ' $b$ ' is the smallest diameter. Lung computed tomography (CT) (Inveon, Siemens) and Carestream Multispectral program (Carestream, NY, USA) were also performed on day 14 after TAE to monitor tumor metastasis. On day 14 after TAE, 15 randomly selected rats from each group were sacrificed. Liver, lung, kidney, and spleen were collected for further investigation, and tumor growth and pulmonary metastasis were assessed. The remaining six rats from each group were kept for survival analysis. Survival time was defined as the interval between the day of inoculation and the day of death.

\section{Detection of CTCs by flow cytometry}

A whole blood sample was collected from anesthetized rats (at least six rats in each group for each time point) from the tail vein. Peripheral blood mononuclear cells (PBMCs, including CTCs) were enriched by Ficoll-Paque density centrifugation separation. ${ }^{16}$ PBMCs from rats without tumors were used as negative controls to exclude the influence of autofluorescence and to select the appropriate gate in flow cytometry. $\mathrm{GFP}^{+} \mathrm{CTC}$ were then counted by using the selected gate. Flow cytometric analysis was performed using an FACS Arial (BD Biosciences, San Jose, CA, USA).

\section{Pathological examination}

Liver, lung, kidney, and spleen were harvested from rats and tested by pathological examination to assess metastasis. Tissues were fixed by formalin, embedded, and sliced into $5 \mu \mathrm{m}$ thick sections. Tissue sections were stained with hematoxylin and eosin (H\&E) using the standard protocol. The maximum coronal plane of left and right lung sections were examined by H\&E staining to assess the presence of metastasis. ${ }^{19}$ The number of lung nodules and other metastatic lesions was counted under an optical microscope.

\section{Western blot analysis}

The expression of E-cadherin (1:1,000), N-cadherin (1:500), vimentin $(1: 500), \operatorname{HIF}-1 \alpha(1: 1,000)$ and GAPDH $(1: 2,000)$ 
was detected by immunoblotting as previously described. ${ }^{14}$ The protein concentration from tumor tissues was determined by BCA Protein Assay Kit (Beyotime, Shanghai, People's Republic of China). Equal amounts of total protein were separated by $10 \%$ SDS-PAGE at $100 \mathrm{~V}$, and transferred to polyvinylidene difluoride (PVDF) membrane (Millipore, Bedford, MA, USA). The membrane was blocked in $10 \%$ milk and probed with the primary antibodies overnight ( 16 hours) at $4^{\circ} \mathrm{C}$. Membrane was then washed and incubated with horseradish peroxidase (HRP)-conjugated secondary antibodies (goat antimouse or goat anti-rabbit immunoglobulin [Ig]G antibody) (1:5,000, Dingguo, Beijing, People's Republic of China) for 1 hour at $37^{\circ} \mathrm{C}$. The protein band was visualized and quantified by densitometry using Image Lab 3.0 on a Molecular Imager ChemiDoc XRS imaging system (Bio-Rad, Hercules, CA, USA). The relative density of each band was determined by the ratio to that of the internal control, GAPDH.

\section{Immunohistochemistry}

Using a standard protocol, ${ }^{19}$ tumor sections were used for immunostaining of E-cadherin $(1: 1,000)$, N-cadherin (1:500), vimentin $(1: 1,000)$, and HIF-1 $\alpha$ (1:500). After deparaffinization and rehydration, slides were treated with antigen retrieval (Dako, Carpinteria, CA, USA) at $97^{\circ} \mathrm{C}$ for 45 minutes. Sections were then stained with appropriate primary antibodies and corresponding secondary antibodies following the Catalyzed Signal Amplification System (Dako). Nuclei were counterstained with hematoxylin. Two or three fields (×200 magnification) from each slide were counted to determine the staining frequency of HIF-1 $\alpha$, E-cadherin, $\mathrm{N}$-cadherin, and vimentin. A total of 15 fields in each group were counted. The expression of these proteins was assessed by the percentage of immunoreactive cells in a certain area of neoplastic cells.

\section{Statistics and data analyses}

Data were expressed as mean \pm standard deviation (SD). The results of in vitro cell invasion assays, tumor volume, and the expression of E-cadherin, N-cadherin, vimentin, and HIF-1 $\alpha$ between the two groups were analyzed by Student's $t$-test. Fisher's exact test or the Wilcoxon rank-sum test was used to compare the incidence of intrahepatic and lung metastasis, and the survival was examined via the Kaplan-Meier method with a log rank test between two groups. $P<0.05$ was considered statistically significant. Analyses were performed by using the SPSS software version 19 (SPSS, Chicago, IL, USA).

\section{Results}

\section{Hypoxia promotes cell invasion and induces a change of epithelial- mesenchymal transition (EMT)}

In cell invasion assay, McA-RH7777 cells showed an elevated capacity of cell invasion (1.63-fold increase) after $\mathrm{CoCl}_{2}$ treatment $(P=0.000$, Figure 2A). The morphology of $\mathrm{CoCl}_{2}$-treated McA-RH7777 cells was distinct from the typical epithelial orbicular-ovate appearance of untreated McA-RH7777 cells, which showed spindle or irregular shapes with less cell-cell adhesion (Figure 2B). Consistent with phenotypic changes, immunofluorescence (Figure 2C) showed a significant reduction in E-cadherin expression but obvious upregulation of $\mathrm{N}$-cadherin and vimentin expression in $\mathrm{CoCl}_{2}$-treated McA-RH7777 cells. The transition from a dominant E-cadherin distribution on the cell membrane to predominant $\mathrm{N}$-cadherin expression is related to epithelialmesenchymal transition (EMT), which triggers cells with a higher ability to transmigrate basement membranes and stromal tissues.

\section{TAE inhibits tumor growth and prolongs rat survival but promotes liver tumor metastasis}

To investigate the effect of TAE on the metastasis of liver tumor, we established a rat orthotopic liver tumor model with local and distant metastatic potentials (Figure 3A). Before the TAE procedure, the average size of the liver tumor did not significantly differ between the TAE and control groups; the mean diameters of tumors were $8.4 \pm 0.4 \mathrm{~cm}$ and $8.4 \pm 0.5 \mathrm{~cm}$, respectively. After TAE treatment, tumor size was significantly smaller on day 7 and $14(P=0.002$ and $P=0.015$, respectively), with a size of $533.7 \pm 99.9 \mathrm{~mm}^{3}$ and 2,216.1 $\pm 730.6 \mathrm{~mm}^{3}$, compared with the control group $\left(917.8 \pm 210.1 \mathrm{~mm}^{3}\right.$ and 4,041.6 $\left.\pm 1,328.4 \mathrm{~mm}^{3}\right)$ (Figure 3B). The mean survival time in the TAE group was significantly longer than that in the control group $(49.8 \pm 3.5 \mathrm{vs}$ $43.8 \pm 2.1$ days; $P=0.001$ ) (Figure 4 ). An increasing trend was shown with respect to the intrahepatic metastasis from $6.7 \%$ ( 1 of 15 ) in the control group to $20.0 \%$ (3 of $15 ; P=0.598$ ) in the TAE group, and lung metastatic rate from $53.3 \%(8$ of 15$)$ in the control group to $80.0 \%$ (12 of $15 ; P=0.245)$ in the TAE group. Tumor metastasis was not found in other organs in both groups (Table 1).

The mean number of metastatic lung nodules in each rat was significantly increased in the TAE group 


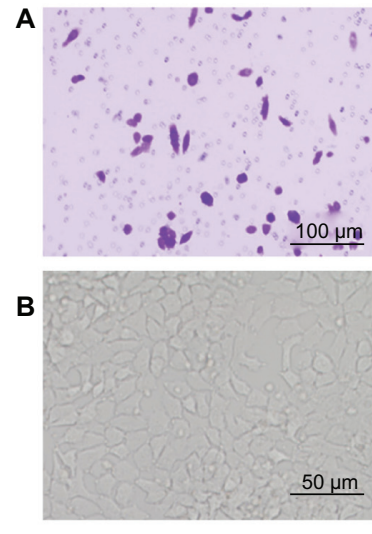

McA-RH7777
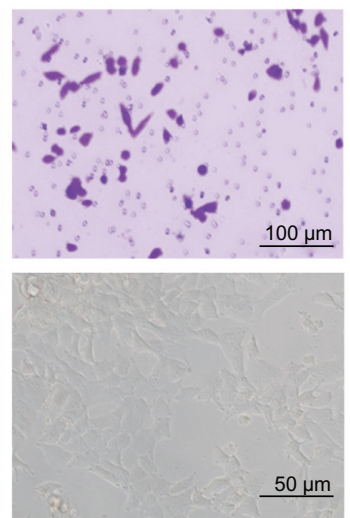

$\mathrm{CoCl}_{2}-\mathrm{McA}-\mathrm{RH} 7777$

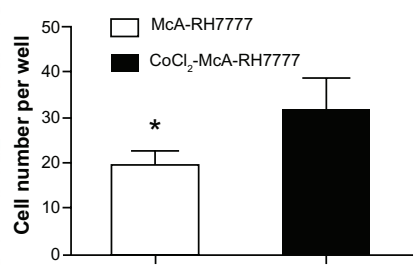

C
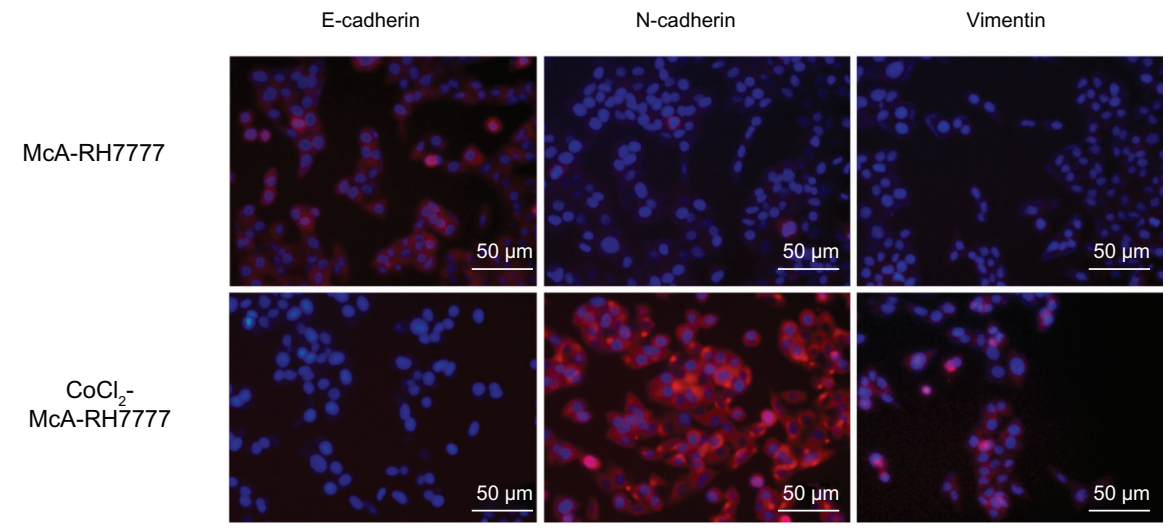

Figure 2 McA-RH7777 cells after $\mathrm{CoCl}_{2}$ treatment exhibited the properties of enhanced invasion and epithelial-mesenchymal transition.

Notes: (A) $\mathrm{CoCl}_{2}$-treated McA-RH7777 cells showed a significant increase in cell invasion capacity compared with $\mathrm{McA}-\mathrm{RH} 7777$ control cells. $* \mathrm{P}<0.05$. (B) CoCl-treated McA-RH7777 cells were morphologically distinct from the typical epithelial appearance of McA-RH7777 cells, showing a spindle shape with less cell-cell adhesion. (C) E-cadherin expression was reduced, but the expression of $\mathrm{N}$-cadherin and vimentin was increased in $\mathrm{CoCl}_{2}$-treated $\mathrm{McA}-\mathrm{RH} 7777$ cells relative to McA-RH7777 cells; this was examined by immunofluorescence.

Abbreviation: $\mathrm{CoCl}_{2}$, cobalt chloride.

compared with that in the control group $(23.3 \pm 12.8 \mathrm{vs}$ $12.4 \pm 5.9 ; P=0.047$ ) (Table 1 and Figure 5A). Intrahepatic and lung metastases were detected by the imaging system and further confirmed by histology examination (Figure 5B).

\section{TAE treatment significantly increases the number of CTCs}

To explore the underlying mechanism of TAE on promoting tumor metastasis, we examined the population of CTCs at different time points after treatment. Before the treatment, the number of CTCs did not obviously differ between the TAE group and the control group. The number of circulating CTCs gradually increased after the treatment in the TAE group. The average level of CTCs was significantly increased by TAE treatment from day 7 to day 21 (day 7 : 52 vs $73 / 10^{6}$ PBMC cells, $P=0.000$; day 14 : 93 vs $189 / 10^{6}$
PBMC cells, $P=0.000$; day 21: 285 vs 593/10 $\mathrm{PBMC}$ cells, $P=0.000$ ) (Figure 6). In both TAE and control groups, the number of CTCs was higher in the rats with metastasis than without metastasis. Therefore, in our tumor model, the number of CTCs corresponds to advanced metastatic tumor and the change of EMT.

\section{TAE upregulates the expression of HIF-I $\alpha, \mathrm{N}$-cadherin, and vimentin but downregulates the expression of E-cadherin in liver tumor tissues}

To explore the underlying mechanism of TAE on increasing CTCs, the changes in molecules in liver tumor tissues was investigated. Here, we also observed the changes of EMT in vivo (Figure 7A). In the TAE group, E-cadherin expression was markedly reduced, especially at the invasive front, while the mesenchymal markers $\mathrm{N}$-cadherin and vimentin 

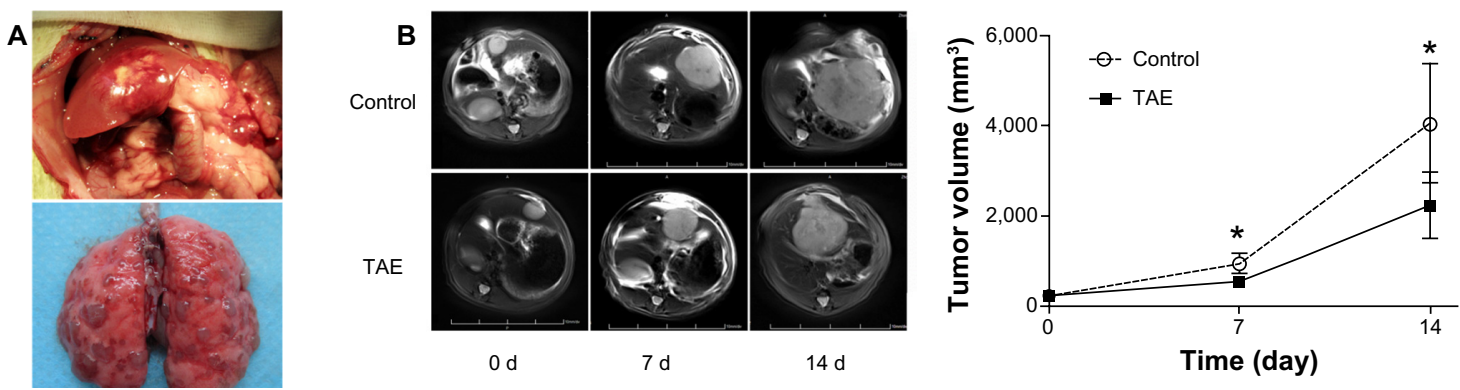

Figure 3 Orthotopic liver tumor model and the comparison of tumor size between the TAE group and the control group.

Notes: (A) Orthotopic liver tumor model with metastatic potential was established in buffalo rats. (B) Tumor size in the TAE group was significantly smaller on days 7 and 14 compared with that in the control group; $* P<0.05$.

Abbreviation: TAE, transcatheter arterial embolization.

were significantly upregulated. Nuclear expression of HIF-1 $\alpha$ was also significantly increased in the TAE-treated tumors compared with the control group. Moreover, the mean percentages of E-cadherin-, N-cadherin-, vimentin-, and HIF- $1 \alpha$-positive cells in the control group and the TAE group were $33.33 \pm 7.24$ vs $13.00 \pm 7.27,42.00 \pm 8.62$ vs $71.33 \pm 9.16,54.00 \pm 9.86$ vs $82.00 \pm 9.41$, and $43.33 \pm 11.13$ vs $81.33 \pm 9.72$, respectively ( $P=0.000$ for all). To verify the results of immunostaining, the relative expression of these proteins was measured by Western blot analysis (Figure 7B). The relative expression of E-cadherin, $\mathrm{N}$-cadherin, vimentin, and HIF- $1 \alpha$ in the control group and the TAE group was $0.74 \pm 0.21$ vs $0.39 \pm 0.17,0.20 \pm 0.08$ vs $0.35 \pm 0.10$, $0.49 \pm 0.10$ vs $0.75 \pm 0.12$, and $0.21 \pm 0.11$ vs $0.79 \pm 0.14$, respectively ( $P=0.000$ for all).

\section{Discussion}

The side effects of TAE treatment in HCC patients have been studied; however, little is known about the underlying mechanism. In the present study, we showed that in vitro exposure of McA-RH7777 cells to hypoxic conditions by $\mathrm{CoCl}_{2}$ led to increased cell invasion. In addition to increasing the incidence of tumor metastasis, TAE also significantly increased the number of metastatic tumor nodules in the lung at 2 weeks after TAE treatment; these findings are consistent with previous studies. ${ }^{5,6}$ These results indicate that TAE treatment may increase the metastatic potential of HCC cells, which is a possible side effect of TAE treatment. This model effectively mimicked the clinical situation of liver tumor metastasis after TAE.

The concept that metastasis is a hallmark of malignancy has been widely understood. It is commonly accepted that the

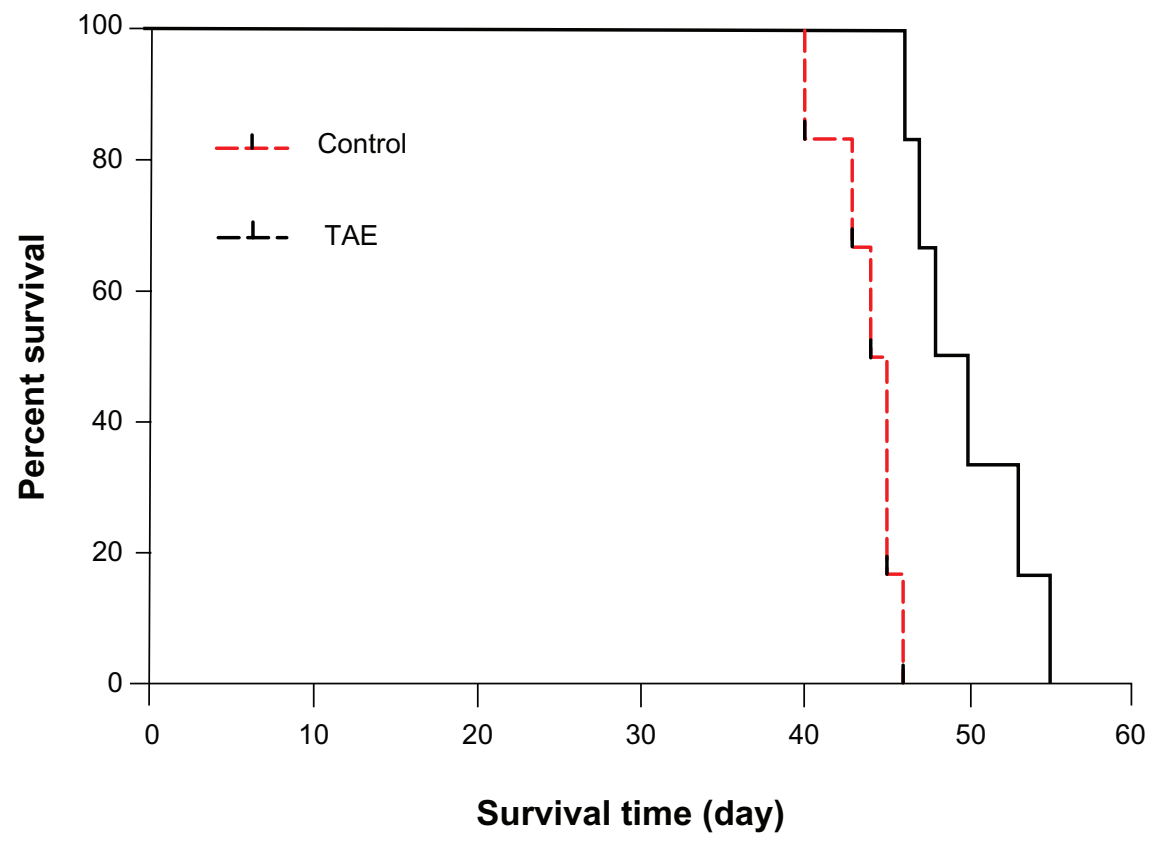

Figure 4 The mean survival time in both groups. The mean survival time in the TAE group was significantly longer than that in the control group. Abbreviation: TAE, transcatheter arterial embolization. 
Table I Comparison of tumor metastasis after transcatheter arterial embolization

\begin{tabular}{llll}
\hline & Control group & TAE group & P-value \\
\hline Lung metastasis & & & \\
$\quad$ Metastasis ratio & $8 / 15(53.3 \%)$ & $12 / 15(80.0 \%)$ & 0.245 \\
$\quad \begin{array}{l}\text { Number } \\
\text { (tumor nodules)/rat }\end{array}$ & $12.4 \pm 5.9$ & $23.3 \pm 12.8$ & 0.047 \\
$\begin{array}{l}\text { Intrahepatic metastasis } \\
\quad \text { Metastasis ratio }\end{array}$ & $1 / 15(6.7 \%)$ & $3 / 15(20.0 \%)$ & 0.598 \\
\hline
\end{tabular}

Abbreviation: TAE, transcatheter arterial embolization.

existence of 'seed' can establish a new tumor in the 'soil' of distant organs. This 'seed and soil' hypothesis was first proposed in the late 19th century, and described the mechanism by which cancer spreads or metastasizes throughout the body and verified the existence of CTCs (seed). Indeed, CTCs that play an important role in tumor growth and metastasis have recently attracted much attention as potential biomarkers and as an ideal model for studying the process of metastasis..$^{21,24,25}$
A decrease in CTCs can impair tumor growth and inhibit metastasis. Furthermore, CTCs play a major role in tumor progression from micrometastases to macrometastases. ${ }^{22}$ Therefore, it is worthwhile studying whether TAE promotes liver tumor metastasis by increasing CTCs, and exploring the underlying mechanism.

In this study, we first demonstrated that TAE promoted liver tumor metastasis by increasing CTCs in an orthotopic rat liver tumor model. CTCs cannot remain in circulation for long in solid tumors. CTCs reflect the combination of two processes: entrance and depletion. The depletion of CTCs may be caused by apoptosis and immune system clearance. The appearance and increase of CTCs depend on the malignancy of the original tumor and the tumor size. ${ }^{26}$ Therefore, the effect of TAE on promoting liver tumor metastasis is probably due to the increase of CTCs, which leads to increasing entrance

A
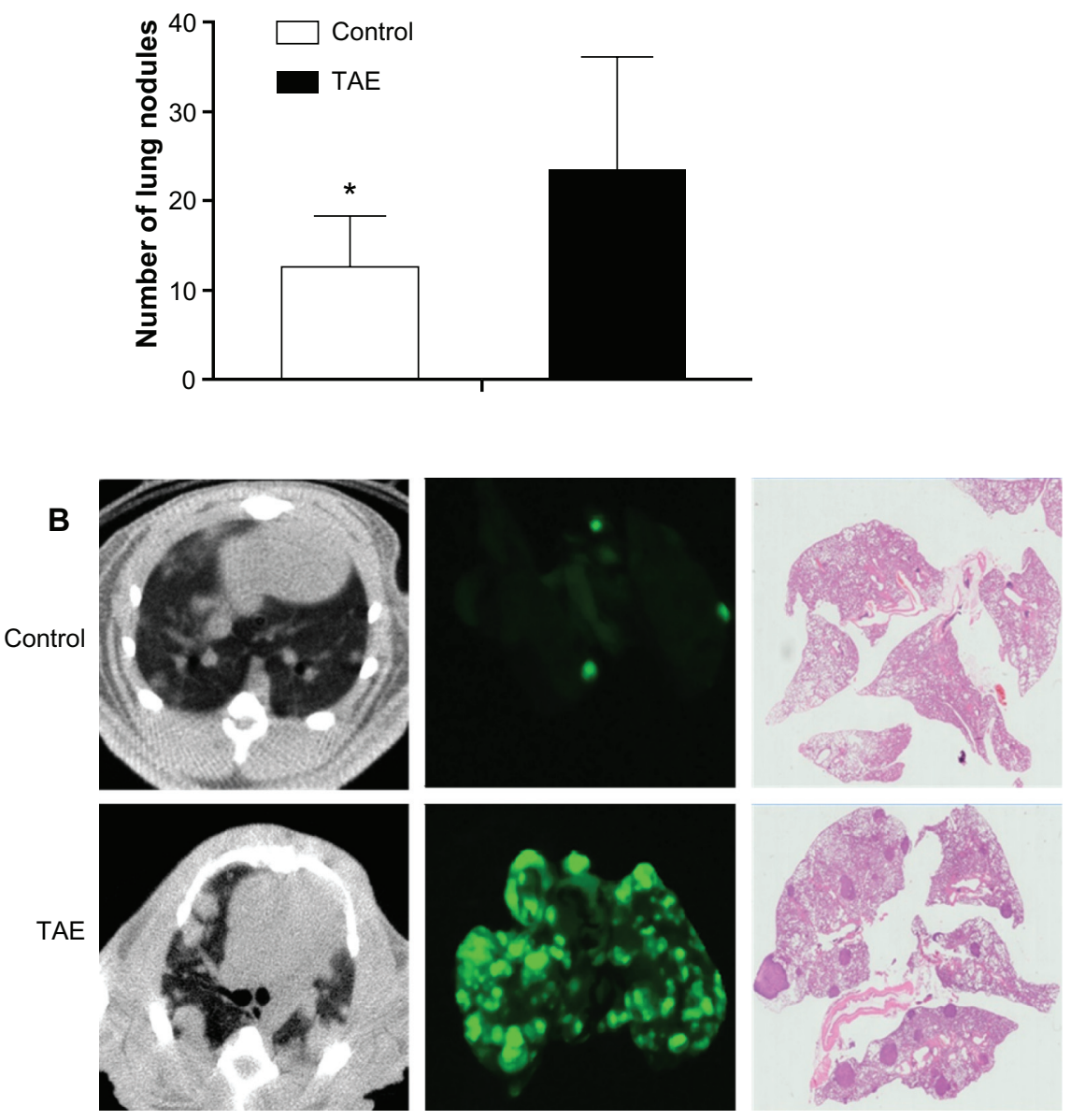

Figure $\mathbf{5}$ Lung metastasis was detected by the imaging system and further confirmed by histology examination.

Notes: (A) The mean number of metastatic lung nodules in both groups; $* P<0.05$. (B) Lung CT and Carestream Multispectral program were performed on day I4 after TAE to monitor tumor metastasis between two groups. The left and right lung sections of the maximum coronal plane were examined by $\mathrm{H} \& \mathrm{E}$ staining to identify the presence of metastasis and count the number of lung nodules under an optical microscope.

Abbreviations: CT, computed tomography; H\&E, hematoxylin and eosin; TAE, transcatheter arterial embolization. 


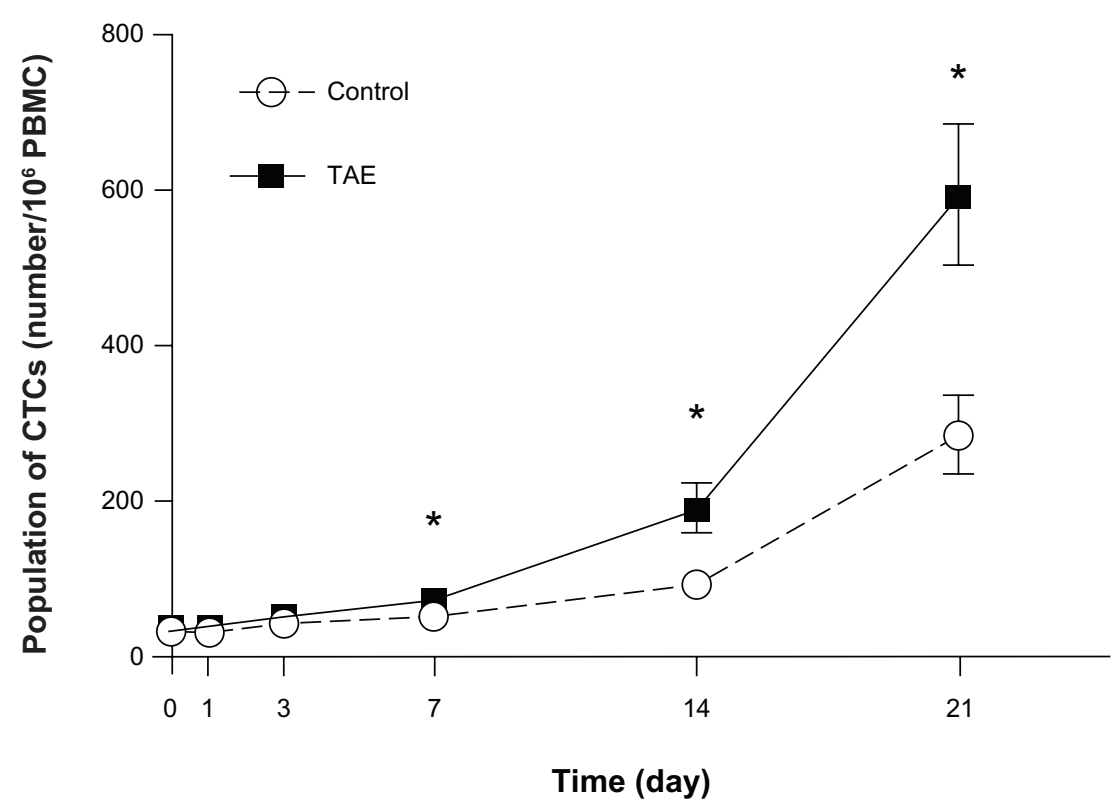

Figure 6 The average level of CTCs was significantly increased in the TAE group from day 7 to day 21 compared with control group. Note: $* P<0.05$.

Abbreviations: CTC, circulating tumor cell; PBMC, peripheral blood mononuclear cells; TAE, transcatheter arterial embolization.

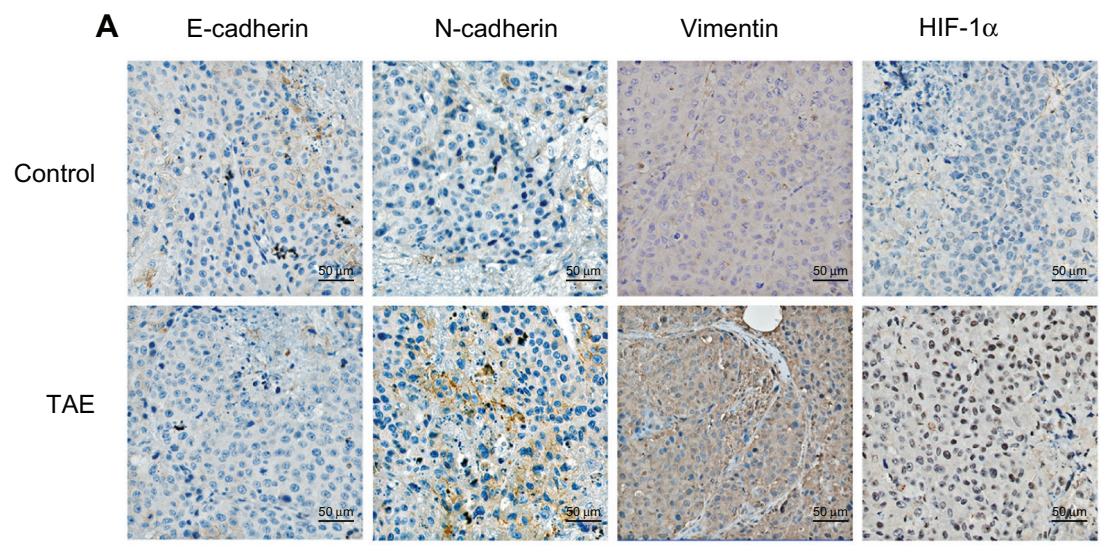

B

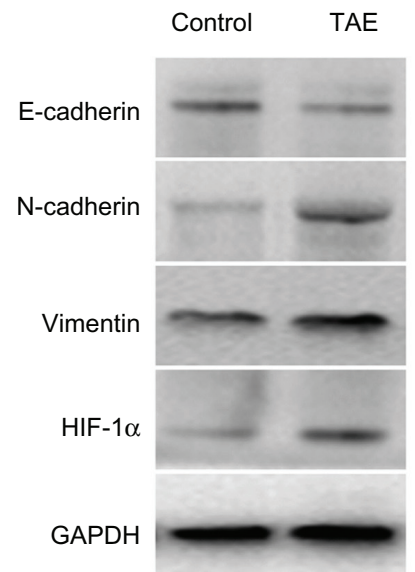

Figure 7 The effect of TAE on the changes of EMT in vivo.

Notes: (A) TAE reduced the expression of E-cadherin but significantly upregulated the expression of $\mathrm{N}$-cadherin, vimentin, and HIF-I $\alpha$ by immunohistochemical analysis. (B) The results of immunohistochemical analysis were confirmed by Western blot analysis.

Abbreviations: EMT, epithelial-mesenchymal transition; GAPDH, glyceraldehyde 3-phosphate dehydrogenase; HIF, hypoxia-inducible factor; TAE, transcatheter arterial embolization. 
and/or decreasing depletion. It is not clear whether the increased metastatic potential of residual HCC neutralizes the effect of TAE treatment; the findings of the present study demonstrate that the increased metastatic potential of residual HCC cells may limit the therapeutic benefit of TAE treatment.

As we know, TAE can enhance intra-tumoral hypoxia that upregulates the expression of HIF-1 $\alpha$. Several studies have shown that HIF-1 $\alpha$ can promote tumor metastases through the acquisition of EMT features, thus leading to increased carcinogenesis and metastasis, stem cell features, invasion, tumor recurrence, etc. To investigate the underlying mechanism of TAE on the increase of CTCs, the expression levels of HIF- $1 \alpha$ and several EMT marker proteins were analyzed. Our results indicated that TAE significantly upregulated the protein levels of $\mathrm{N}$-cadherin, vimentin, and HIF-1 $\alpha$ but downregulated E-cadherin expression. These data thus suggest that EMT-derived cells enhance the metastatic potential of primary tumors.

The EMT process is currently believed to play a key role in CTCs escaping from the primary tumor tissue, invading the surrounding stroma, intravasation of the vasculature, and thus developing metastasis. The contribution of EMT to the generation of CTCs is hitherto largely unexplored. The EMT-derived cells display resistance to apoptotic signals, which help CTCs survive in the bloodstream. ${ }^{27}$ A recent report suggested that a direct link might exist between the EMT and the acquisition of stem cell properties ${ }^{28-30}$ Furthermore, cells undergoing EMT could develop resistance against conventional chemotherapy and possibly escape immune surveillance. In this context, EMT is considered to be one of the factors that enhance metastatic potential.

Therefore, we speculate that EMT induced by TAE may contribute to the increase of CTCs by increasing entrance and decreasing depletion. This model explored the role of EMT in the whole process of metastasis. These results, together with enhanced pulmonary metastasis rates in the TAE group, highlight the close relationship among TAE treatment, EMT, CTCs, and metastasis. Further studies are needed to clarify the detailed mechanisms.

\section{Conclusion}

TAE promotes liver tumor metastasis through the acquisition of EMT features and by increasing the number of CTCs, suggesting that it is necessary to develop a promising adjuvant therapy for TAE treatment to reduce the risk of tumor recurrence and metastasis in HCC patients.

\section{Acknowledgment}

This work was supported by grant number 81171432 from the National Natural Sciences Foundation of China.

\section{Disclosure}

The authors report no conflicts of interest in this work.

\section{References}

1. Jemal A, Bray F, Center MM, Ferlay J, Ward E, Forman D. Global cancer statistics. CA Cancer J Clin. 2011;61(2):69-90.

2. Llovet JM, Real MI, Montaña X, et al. Arterial embolisation or chemoembolisation versus symptomatic treatment in patients with unresectable hepatocellular carcinoma: a randomised controlled trial. Lancet. 2002;359(9319):1734-1739.

3. Lo GH. Updated management of hepatocellular carcinoma Hepatology. 2011;54(3):1113.

4. Bruix J, Sherman M; American Association for the Study of Liver Diseases. Management of hepatocellular carcinoma: an update. Hepatology. 2011;53(3):1020-1022.

5. Lin SC, Shih SC, Kao CR, Chou SY. Transcatheter arterial embolization treatment in patients with hepatocellular carcinoma and risk of pulmonary metastasis. World J Gastroenterol. 2003;9(6):1208-1211.

6. Chen C, Wang J, Liu R, Qian S. RNA interference of hypoxia-inducible factor-1 alpha improves the effects of transcatheter arterial embolization in rat liver tumors. Tumour Biol. 2012;33(4):1095-1103.

7. Apte S, Chin FT, Graves EE. Molecular imaging of hypoxia: strategies for probe design and application. Curr Org Synth. 2011;8(4):593-603.

8. Koi M, Boland CR. Tumor hypoxia and genetic alterations in sporadic cancers. J Obstet Gynaecol Res. 2011;37(2):85-98.

9. Rofstad EK, Gaustad JV, Egeland TA, Mathiesen B, Galappathi K. Tumors exposed to acute cyclic hypoxic stress show enhanced angiogenesis, perfusion and metastatic dissemination. Int J Cancer. 2010; 127(7):1535-1546.

10. Kim Y, Lin Q, Glazer PM, Yun Z. Hypoxic tumor microenvironment and cancer cell differentiation. Curr Mol Med. 2009;9(4):425-434.

11. Lianidou ES. Circulating tumor cells: new challenges ahead. Clin Chem. 2012;58(5):805-807.

12. Scatton O, Chiappini F, Liu XH, et al. Generation and modulation of hepatocellular carcinoma circulating cells: a new experimental model. J Surg Res. 2008;150(2):183-189.

13. Sleeman JP, Nazarenko I, Thiele W. Do all roads lead to Rome? Routes to metastasis development. Int J Cancer. 2011;128(11):2511-2526.

14. Cristofanilli M, Budd GT, Ellis MJ, et al. Circulating tumor cells, disease progression, and survival in metastatic breast cancer. $N$ Engl $J$ Med. 2004;351(8):781-791.

15. Cen P, Ni X, Yang J, Graham DY, Li M. Circulating tumor cells in the diagnosis and management of pancreatic cancer. Biochim Biophys Acta. 2012;1826(2):350-356.

16. Kim MY, Oskarsson T, Acharyya S, et al. Tumor self-seeding by circulating cancer cells. Cell. 2009;139(7):1315-1326.

17. Ho JW, Pang RW, Lau C, et al. Significance of circulating endothelial progenitor cells in hepatocellular carcinoma. Hepatology. 2006;44(4): 836-843.

18. Yan J, Zhuo S, Chen G, et al. Use of multiphoton microscopy to diagnose liver cancer and lung metastasis in an orthotopic rat model. Scanning. 2012;34(4):271-277.

19. Wu LJ, Pan YD, Pei XY, et al. Capturing circulating tumor cells of hepatocellular carcinoma. Cancer Lett. 2012;326(1):17-22.

20. $\mathrm{Xu} \mathrm{W}, \mathrm{Cao} \mathrm{L}$, Chen $\mathrm{L}$, et al. Isolation of circulating tumor cells in patients with hepatocellular carcinoma using a novel cell separation strategy. Clin Cancer Res. 2011;17(11):3783-3793.

21. Fan ST, Yang ZF, Ho DW, Ng MN, Yu WC, Wong J. Prediction of posthepatectomy recurrence of hepatocellular carcinoma by circulating cancer stem cells: a prospective study. Ann Surg. 2011;254(4):569-576. 
22. Gao D, Nolan DJ, Mellick AS, Bambino K, McDonnell K, Mittal V. Endothelial progenitor cells control the angiogenic switch in mouse lung metastasis. Science. 2008;319(5860):195-198.

23. Kan Z, Kobayashi S, Phongkitkarun S, Charnsangavej C. Functional CT quantification of tumor perfusion after transhepatic arterial embolization in a rat model. Radiology. 2005;237(1):144-150.

24. Paterlini-Brechot P, Benali NL. Circulating tumor cells (CTC) detection: clinical impact and future directions. Cancer Lett. 2007;253(2): 180-204.

25. O'Flaherty JD, Gray S, Richard D, et al. Circulating tumour cells, their role in metastasis and their clinical utility in lung cancer. Lung Cancer. 2012;76(1):19-25.

26. Fan ZC, Yan J, Liu GD, et al. Real-time monitoring of rare circulating hepatocellular carcinoma cells in an orthotopic model by in vivo flow cytometry assesses resection on metastasis. Cancer Res. 2012;72(10):2683-2691.
27. Bonnomet A, Brysse A, Tachsidis A, et al. Epithelial-to-mesenchymal transitions and circulating tumor cells. J Mammary Gland Biol Neoplasia. 2010;15(2):261-273.

28. Aktas B, Tewes M, Fehm T, Hauch S, Kimmig R, Kasimir-Bauer S. Stem cell and epithelial-mesenchymal transition markers are frequently overexpressed in circulating tumor cells of metastatic breast cancer patients. Breast Cancer Res. 2009;11(4):R46.

29. Mani SA, Yang J, Brooks M, et al. Mesenchyme Forkhead 1 (FOXC2) plays a key role in metastasis and is associated with aggressive basal-like breast cancers. Proc Natl Acad Sci U S A. 2007;104(24): 10069-10074.

30. Mani SA, Guo W, Liao MJ, et al. The epithelial-mesenchymal transition generates cells with properties of stem cells. Cell. 2008;133(4): 704-715.
OncoTargets and Therapy

\section{Publish your work in this journal}

OncoTargets and Therapy is an international, peer-reviewed, open access journal focusing on the pathological basis of all cancers, potential targets for therapy and treatment protocols employed to improve the management of cancer patients. The journal also focuses on the impact of management programs and new therapeutic agents and protocols on

\section{Dovepress}

patient perspectives such as quality of life, adherence and satisfaction. The manuscript management system is completely online and includes a very quick and fair peer-review system, which is all easy to use. Visit http://www.dovepress.com/testimonials.php to read real quotes from published authors. 